\title{
Genetic aspects of primary hyperaldosteronism
}

\author{
Weronika Korzyńska ${ }^{1, A-D}$, Anna Jodkowska, ${ }^{1, A, C-E}$, Katarzyna Gosławska ${ }^{1, B}$, \\ Katarzyna Bogunia-Kubik ${ }^{1,2, A, E, F}$, Grzegorz Mazur ${ }^{1, F}$ \\ 1 Department and Clinic of Internal and Occupational Diseases and Hypertension, Wroclaw Medical University, Poland \\ ${ }^{2}$ Laboratory of Clinical Immunogenetics and Pharmacogenetics, Institute of Immunology and Experimental Therapy, Polish Academy of Sciences, Wrocław, Poland \\ $A$ - research concept and design; $B$ - collection and/or assembly of data; $C$ - data analysis and interpretation; \\ $\mathrm{D}$ - writing the article; $\mathrm{E}$ - critical revision of the article; $\mathrm{F}$ - final approval of the article
}

\section{Address for correspondence \\ Anna Jodkowska}

E-mail:anna.jodkowska@umed.wroc.pl

\section{Funding sources}

None declared

\section{Conflict of interest}

None declared

Received on November 6, 2016

Reviewed on December 28, 2016

Accepted on March 8, 2017

\begin{abstract}
Primary hyperaldosteronism (PHA) is the most common form of secondary hypertension of hormonal origin. It affects about 10\% of all hypertensive patients. It is connected with increased morbidity and mortality from cardiovascular diseases (CVD) compared to patients with essential hypertension, at a similar age. Usually, it is an effect of bilateral adrenal hyperplasia (BAH) or aldosterone-producing adenoma (APA), rarer causes of PHA are: unilateral adrenal hyperplasia, aldosterone-producing adrenocortical carcinoma, ectopic aldosterone-producing tumors, and familial hyperaldosteronism (FH). Recent genetic studies have thrown a new light on the pathogenesis of PHA, classifying it as a channelopathy. Several mutations within the ion channels encoding genes have been identified. A possible link between PHA and polymorphism of aldosterone synthase gene and ion channel genes is still being investigated. In this manuscript, we focus on the genetic aspects of PHA, and present an up-to-date compilation of available data with a widened pathogenetic approach.
\end{abstract}

Key words: gene polymorphism, aldosterone, primary hyperaldosteronism, ion channels, angiotensine II

DOI

10.17219/acem/69390

\section{Copyright}

Copyright by Author(s)

This is an article distributed under the terms of the

Creative Commons Attribution Non-Commercial License

(http://creativecommons.org/licenses/by-nc-nd/4.0/) 


\section{Introduction}

Primary hyperaldosteronism (PHA) is the most common cause of secondary hypertension of hormonal origin. It can possibly concern over $10 \%$ of hypertensive patients. ${ }^{1}$ Its occurrence ranges from 8 to $30 \%$ in different reports, depending on the diagnostic criteria and the sample. In the large prospective PAPY study, primary hyperaldosteronism occurred in $11.2 \%$ of newly diagnosed hypertensive patients. ${ }^{2,3}$ The prevalence of PHA augments with an increase of blood pressure value and among patients with resistant hypertension it reaches up to $20 \%$. Primary hyperaldosteronism was first described by Conn in the 1950 s. $^{4}$ The cause of the syndrome is excessive autonomous secretion of aldosterone, which appears to be relatively independent on the renin-angiotensin-aldosterone system (RAAS), adrenocorticotropic hormone $(\mathrm{ACTH})$, and the extracellular concentration of potassium ions. ${ }^{1}$

Primary hyperaldosteronism is not a homogenous pathogenetic syndrome. It frequently occurs as the effect of bilateral adrenal hyperplasia (BAH) known also as idiopathic hyperaldosteronism (IHA) and aldosterone-producing adenoma (APA). Bilateral adrenal hyperplasia and APAs both represent about $95 \%$ cases of PHA (35\% APA and 60\% IHA). ${ }^{2}$ Less often PHA is a result of unilateral adrenal hyperplasia, and in very rare cases it is caused by aldosterone-producing adrenocortical carcinoma, ectopic aldosterone-producing tumors, and familial aldosteronism (FA). ${ }^{2}$

Primary hyperaldosteronism is connected with increased morbidity and mortality from cardiovascular diseases (CVD) compared to patients with essential hypertension of a similar age. ${ }^{5}$ Patients with PHA are at a higher risk of suffering a heart attack, stroke, and atrial fibrillation. ${ }^{5}$ They are affected more frequently by metabolic syndrome as well. ${ }^{6}$

An excess of aldosterone exerts a number of adverse effects on a variety of organs and tissues. It has been reported to increase collagen synthesis, leading to heart muscle fibrosis, concentric remodeling and thickening of the left ventricular wall, as well as to predispose to the deterioration of left ventricular diastolic function. ${ }^{7,8}$ In addition, excess of aldosterone contributes to the damage of the small vessels and to the progress of nephropathy. ${ }^{9}$ Patients with PHA, compared to essential hypertensives, have a higher initial concentration of the $\mathrm{C}$-reactive protein and interleukin $6 .{ }^{10,11}$ Moreover, they are characterized by the increased thickness of the carotid intima-media, increased arterial stiffness, and more advanced vascular endothelial dysfunction than those with essential hypertension. ${ }^{12}$

There is now a growing interest in genetic determination of PHA. Recently, new forms of familial hereditary aldosteronism have been described. A number of somatic mutations connected with the APAs' appearance have been shown as well. In this manuscript we focus on the genetic aspects of primary hyperaldosteronism. We present an upto-date compilation of available data broadened by the pathogenetic approach.

\section{Aldosterone: regulative physiology}

Aldosterone is a steroid hormone synthetized from cholesterol exclusively in the adrenal glomerulosa layer by the aldosterone synthase (CYP11B2). However, there is also some data of its possible synthesis in other human tissues such as the heart, aorta, pulmonary arteries, and brain. ${ }^{13}$ Cholesterol used in aldosterone production may be of various origins, including de novo synthesis from cholesterol esters or the uptake of low-density lipoproteins (LDL). The schematic diagram of aldosterone synthesis is presented in Fig. 1.

Aldosterone synthase (CYP11B2), located exclusively in the adrenal zona glomerulosa, is highly homologous to $11 \beta$-hydroxylase (CYP11B1), which catalyzes the final step of cortisol synthesis and is located in the zona fasciculata of the adrenal gland. Genes encoding CYP11B1 and CYP11B2 are placed on chromosome 8q21-22 in close proximity to each other. This proximity plays an important role in the pathogenesis of familial aldosteronism type $1 .^{14}$

There are many factors known to regulate aldosterone secretion. The most important are considered: angiotensin II, changes of extracellular potassium ion concentration, and adrenocorticotropic hormone (ACTH). ${ }^{15}$

Adrenal glomerulosa layer cells maintain negative resting membrane potential (hyperpolarization), which is primarily retained by activity of ion channels: TASK 1 and 3 channels (TASK; twike-related acid sensitive $\mathrm{K}^{+}$channels) and Kir channels $\left(\mathrm{K}^{+}\right.$inwardly rectifying potassium channels). ${ }^{16}$ Another channels contributing to the maintenance of a negative resting potential are ATPases. $\mathrm{Na}^{+} / \mathrm{K}^{+}$ATPase transports $2 \mathrm{~K}^{+}$ions into the cell and removes $3 \mathrm{Na}^{+}$ions outside the cell and the $\mathrm{Ca}^{2+}$ ATPase removes calcium ions outside the cell, both using the energy from ATP hydrolysis. An important role in the homeostasis of the zona glomerulosa cells is also played by voltage dependent calcium channels: low-voltage activated calcium channels (type $\mathrm{T}$, $\mathrm{Ca}_{\mathrm{v}} 3 . \mathrm{x}$ ) and high-voltage activated calcium channels (type $\mathrm{L}$, $\left.\mathrm{Ca}_{\mathrm{v}} 1 . \mathrm{x}\right)$. Both are closed during the rest cell membrane hyperpolarization and opened during its depolarization, resulting in calcium ion entrance into the cell. ${ }^{16}$

The increase of intracellular calcium concentration plays a crucial role in the majority of signaling pathways of aldosterone production. It enhances the activity of cholesterol ester hydrolase, thereby increasing the bioavailability of cholesterol (a substrate for further aldosterone production), and augments "cytoskeletal" delivery of cholesterol in the vicinity of the outer mitochondrial membrane. Increased concentration of intracellular calcium also enhances mitochondrial oxidative metabolism and the formation of cofactors required for aldosterone synthase (CYP11B2) and cortisol synthase CYP11B1. Calcium ions also enhance the transcription and translation of StAR protein which in turn increases the transport of cholesterol into the internal mitochondrium membrane. ${ }^{16}$ 


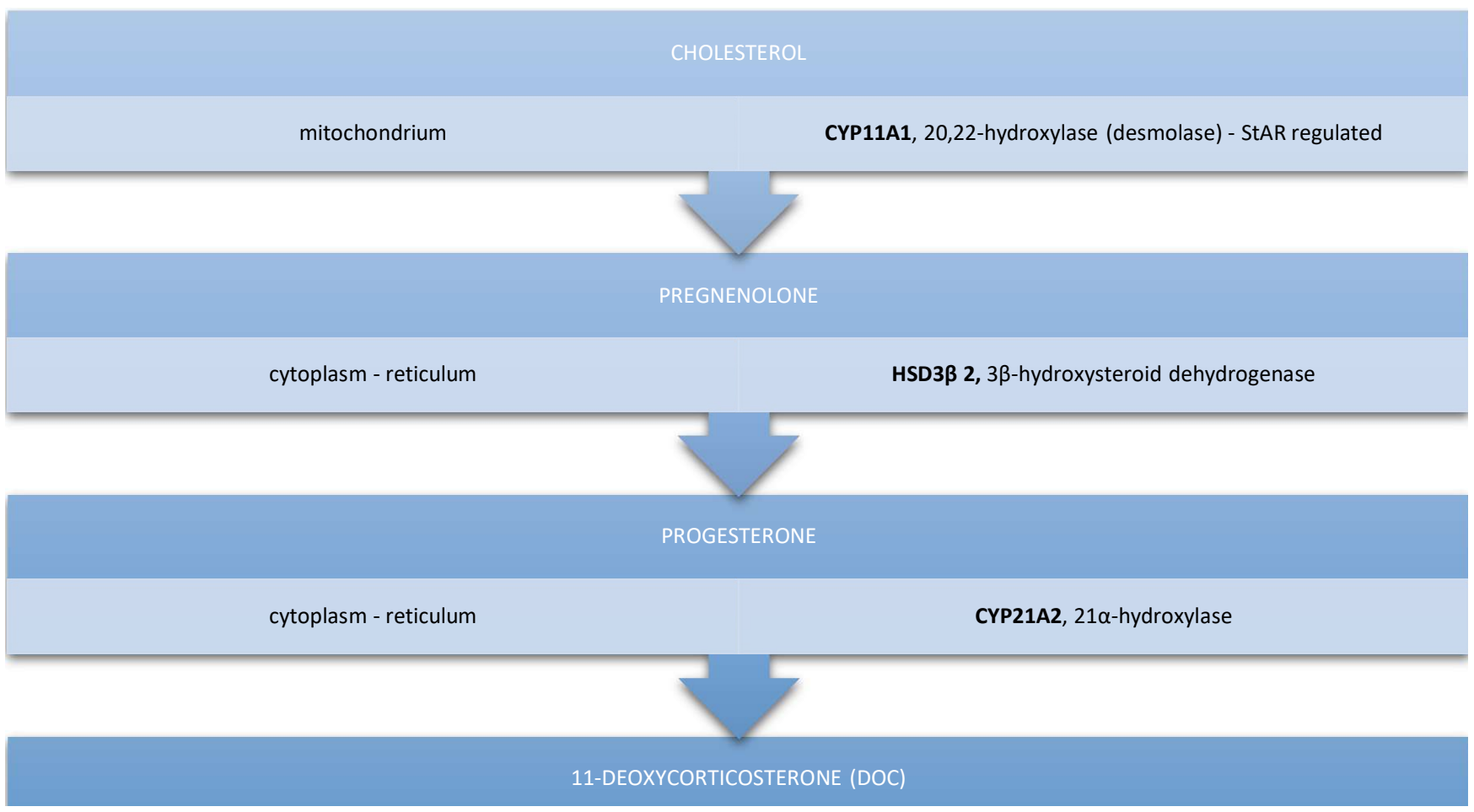

Fig. 1. A schematic diagram of aldosterone synthesis. Cholesterol is transported into the mitochondrium, where it is converted to pregnenolone by enzyme CYP11A1 (desmolase). This step is strictly controlled by the steroidogenic acute regulatory protein (protein StAR). Pregnenolone is then released into the cytoplasm, where it is converted to progesterone by the HSD3B2 dehydrogenase. In a further step progesterone undergoes 21-hydroxylation mediated by CYP21A2, synthetizing 11-deoxycorticosterone (DOC). The last step of the conversion of DOC to aldosterone takes place in the mitochondria of the adrenal zona glomerulosa cells exclusively and it is catalyzed by aldosterone synthase - CYP11B2. It consists of 3 reactions: 11ß-hydroxylation, 18-hydroxylation and 18-methyloxidation

The most important regulator of the aldosterone synthesis is angiotensin II (ATII). It has been shown that chronic stimulation of angiotensin II leads to hyperplasia of the adrenal cells and increased expression of CYP11B2 and then to excessive secretion of aldosterone. ${ }^{13}$ The direct mechanism is shown in Fig. 2. Binding of angiotensin II to angiotensin II type 1 receptor (AT1R) on the adrenal zona glomerulosa cells surface leads to TASK channels, Kir 3.4 channels, and the sodium-potassium pump closure. This results in cell membrane depolarization. ${ }^{15}$ The consequence of a membrane depolarization is the opening of the voltage dependent L- and T-type calcium channels allowing calcium influx into the cell. Calcium mobilization activates a cascade of calcium dependent protein kinases (calcium-calmodulin protein kinases - CaMK), which enhances the expression of aldosterone synthase.

Joining ATII to its AT1R receptor triggers also other mechanisms of aldosterone secretion. It is known to activate phospholipase $\mathrm{C}$, which hydrolyzes phosphatidylinositol 4,5-bisphosphate (PIP2) into inositol 1,4,5-trisphosphate (IP3) and 1,2-diacylglycerol (DAG). These act as follows:

- IP3 stimulates the release of calcium from the endoplasmic reticulum, leading to an increase of intracellular calcium concentration and contributing to the calcium dependent protein kinases activation;
- DAG activates protein kinase C (PKC), which slows the cortisol synthesis pathway by inhibiting transcription of 17-alpha-hydroxylase (CYP17), which competitively promotes aldosterone synthesis, increasing the amount of substrate for it;

- both DAG and its metabolite 12-hydroxyeicosatetraenoic acid (12-HETE) via the protein kinase D (PKD) activate CREB proteins, which further phosphorylate and modulate the expression of StAR protein and lead to increased transcription of aldosterone synthase (CYP11B2). ${ }^{15}$

Another important factor stimulating aldosterone secretion is a change in extracellular potassium ions concentration. The increase in extracellular potassium ion concentration induces the depolarization of the adrenal glomerulosa zone cells. It leads to voltage-dependent calcium channels opening and calcium influx. Calcium mobilization results in the activation of the calcium dependent protein kinases - CaMK, which in turn phosphorylate transcription factors stimulating CYP11B2 transcription. ${ }^{15}$

Among other factors affecting the secretion of aldosterone, adrenocorticotropic hormone should be mentioned. ACTH binds to MC2R (mineralocorticoid type 2 receptor), which activates adenylate cyclase (AC). Adenylate cyclase converts ATP to CAMP, which stimulates the activity of cAMP-dependent kinases including protein kinase 


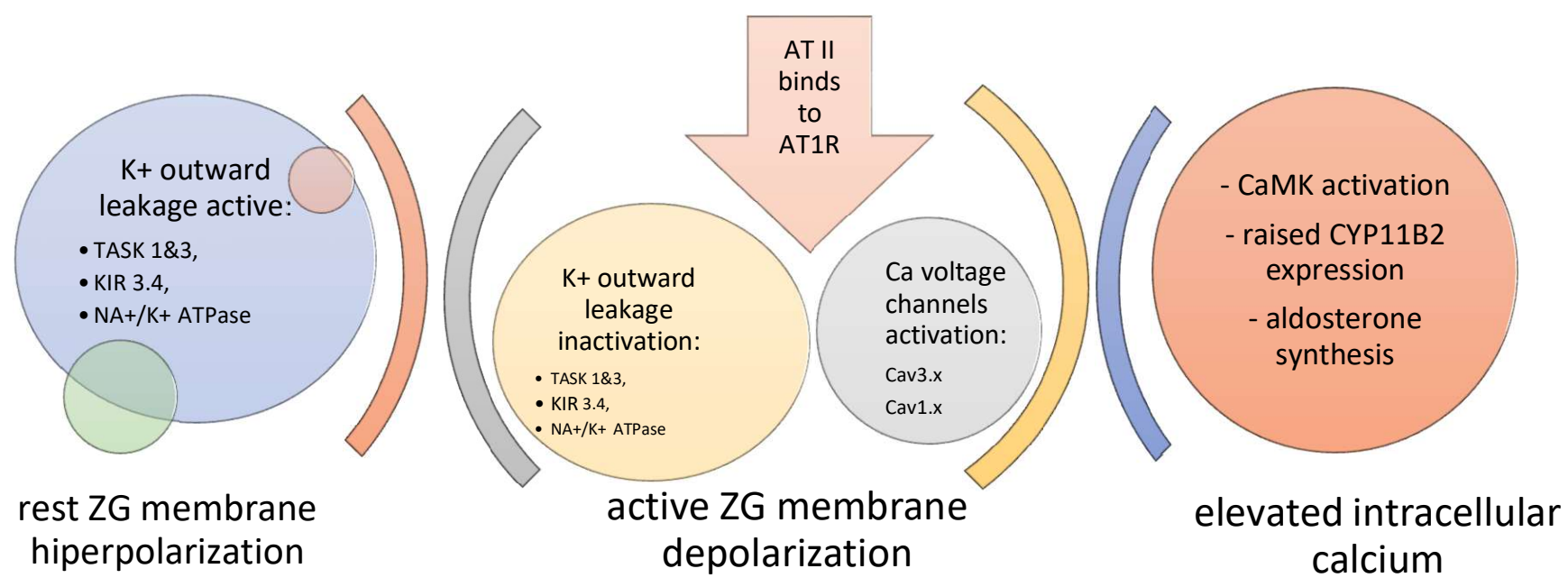

Fig. 2. A schematic diagram of rest and active ion equilibrium in the adrenal zona glomerulosa (ZG) cells. Binding of angiotensin II (AT II) to the AT1R receptor (angiotensin II type 1 receptor) on the adrenal zona glomerulosa cells surface leads to TASK channels, Kir 3.4 channels and the sodium-potassium pump closure. This results in cell membrane depolarization. The consequence of a membrane depolarization is opening of the voltage dependent $L$ and T-type calcium channels (Cav3.x and Cav1.x) allowing calcium influx into the cell. Calcium mobilization activates a cascade of calcium dependent protein kinases (CaMK, calcium-calmodulin protein kinases), which enhances the expression of aldosterone synthase (CYP11B2) and enlarges synthesis of aldosterone

A (PKA). Protein kinase A induces a slow influx of calcium into zona glomerulosa cells by L-type calcium channels; it also regulates the transcriptional activity of CREB proteins, phosphorylates, and activates the StAR protein. The StAR protein increases the transcription of CYP11B2 and aldosterone production. ${ }^{15}$ It has also been shown that, apart from cAMP pathway, ACTH stimulates the aldosterone production through macrophage-derived factor, steroidogenesis-inducing protein and calmidazolium. ${ }^{13}$

\section{Somatic mutations in ion channels and hyperaldosteronism}

Widely conducted genetic studies in the recent few years help to clarify the pathogenesis of PHA and classify the disease as a channelopathy. Pathological, excessive production of aldosterone in PHA is considered to be the result of somatic mutations appearing at different levels of aldosterone synthesis: concerning not only key enzymes but the dysfunction of ion channels as well. The selected mutations in ion channel encoding genes are presented in Table 1.

\section{KCNJ5}

In 2011 Choi et al., sequenced an exome from aldosterone-producing adenomas and identified few somatic KCNJ5 gene mutations, which made a breakthrough in the understanding of the genetics of PHA. ${ }^{17}$ The KCNJ5 gene is located on chromosome 11q24.3 and encodes ion potassium channel Kir3.4. Under physiological conditions, Kir3.4 channel is responsible for the removal of potassium ions out of the cell and the maintenance of the membrane hyperpolarization. Together with ion-channel Kir3.1, it may form a high-active heterotetramer or less-active homotetramer. Choi et al. investigated the material from
22 APA patients and identified 2 types of somatic point mutations: G151R p.Gly151Arg, and L168R p.Leu168Arg (Table 1). Glycine - G151 is the first glycine belonging to the GYG motif (glycine-tyrosine-glycine) in the coding region of the potassium channel selectivity filter. ${ }^{17}$ The side chain of leucine - L168 closely adheres to the side chain of tyrosine in mentioned GYG motif. ${ }^{17}$ The described mutations cause a loss of Kir3.4 filter selectivity, which results in intracellular sodium current and the depolarization of the cell membrane independently on AT receptor binding. Depolarization triggers an opening of the voltage-gated calcium channels and the appearance of the intracellular calcium influx. The increase in the intracellular calcium concentration initializes a cascade of mechanisms, leading to increased aldosterone production and adrenal zona glomerulosa cells proliferation as described previously. Another somatic point mutation in KCNJ5 gene - p.Thy158Ala (T158A), which was identified by Mulatero et al. in 2012. ${ }^{18}$ This mutation was earlier investigated by Choi et al. as a germline mutation in familial hyperaldosteronism type $3 .{ }^{17}$ It similarly leads to a loss of selectivity filter, sodium current influx, and cell membrane depolarization. So far, several somatic mutations in KCNJ5 have been described - p.Ile157del, p.Glu145Gln (E145Q), and p.Trp126Arg (W126R); however, they occur extremely rarely. ${ }^{19-21}$ Recent research allowed us to identify some further mutations of KCNJ5. ${ }^{22-24}$ Scholl et al. described the presence of point mutations: I157K, F154C, and insertions I150_G151insM and I144_E145insAI, located within the coding region of the ion channel selectivity filter as well. ${ }^{22}$ Zheng et al. described different mutation c.445446insGAA, p.T148-T149insR, while Wang et al. identified 3 new mutations - a point mutation E147Q, an insertion mutation c.448-449insCAACAACCA and duplication G153_G164dup. ${ }^{23-24}$ Current data allows us to estimate the prevalence of somatic KCNJ5 gene mutations among 
Table 1. Mutations detected in patients with primary aldosteronism

\begin{tabular}{|c|c|c|c|}
\hline Gene & Description and protein & Chromosome & Mutation \\
\hline KCNJ5 & $\begin{array}{l}\text { potassium voltage-gated channel subfamily J member } 5 \\
\text { known as: KIR3.4, GIRK4, CIR, KATP1, LQT13 }\end{array}$ & 11q24.3. & $\begin{array}{c}\text { somatic: } \\
\text { G151R p.Gly151Arg } \\
\text { L168R p.Leu168Arg } \\
\text { T158A p.Thy158Ala } \\
\text { p.lle157del } \\
\text { E145Q p.Glu145GIn } \\
\text { W126R p.Trp126Arg } \\
\text { I157K p.lle157Lys } \\
\text { F154C p.Phe154Cys } \\
\text { I150_G151insM } \\
\text { I144_E145insAl } \\
\text { E147Q p.Glu147GIn } \\
\text { p.G153_G164dup, c.457-492dupG-G, } \\
\text { C.448-449insCAACAACCA } \\
\text { germline: } \\
\text { T158A p.Thy158Ala } \\
\text { G151R p.Gly151Arg } \\
\text { G151E p.Gly151Glu } \\
\text { I157S p.lle157Ser }\end{array}$ \\
\hline ATP1A1 & ATPase $\mathrm{Na}^{+} / \mathrm{K}^{+}$transporting subunit alpha 1 & $1 \mathrm{p} 21$ & $\begin{array}{l}\text { somatic: } \\
\text { L104R p.Leu104Arg } \\
\text { V332G p.Val332Gly } \\
\text { p.Phe100_Leu104del } \\
\text { G99R p.Gly99Arg } \\
\text { p.GluGluThrAla963Ser }\end{array}$ \\
\hline ATP2B3 & $\begin{array}{c}\text { ATPase plasma membrane } \mathrm{Ca}^{2+} \text { transporting } 3 \\
\text { known as: CLA2, OPCA, PMCA3, SCAX1, CFAP39, PMCA3a }\end{array}$ & Xq28 & $\begin{array}{c}\text { somatic: } \\
\text { p.Leu425_Val426del } \\
\text { p.Val426_pVal427del } \\
\text { p.Arg428-Val429del c.1281_1286delGGCTGT } \\
\text { V426G_V427Q_A428_L433del } \\
\text { p.Val424_Leu425del }\end{array}$ \\
\hline CACNAID & $\begin{array}{c}\text { calcium voltage-gated channel subunit alpha } 1 \text { D } \\
\text { known as: Cav1.3, CACH3, CACN4, PASNA, CCHL1A2, } \\
\text { CACNL1A2 }\end{array}$ & $3 p 14.3$ & $\begin{array}{l}\text { somatic: } \\
\text { G403R p.Gly403Arg } \\
\text { 1770M p.lle770Met } \\
\text { I750M p.lle750Met } \\
\text { F747L p.Phe747Leu } \\
\text { R990H p.Arg990His } \\
\text { P1336R p.Pro1336Arg } \\
\text { V259D p.Val259Asp } \\
\text { V748I p.Val748Ile } \\
\text { germline: } \\
\text { G403R p.Gly403Asp } \\
\text { I770M p.lle770Met }\end{array}$ \\
\hline CACNAIH & $\begin{array}{l}\text { calcium voltage-gated channel subunit alpha } 1 \mathrm{H} \\
\text { known as: Ca,3.2, ECA6, ElG6, HALD4, CACNA1HB }\end{array}$ & $16 p 13.3$ & $\begin{array}{l}\text { germline: } \\
\text { M1549V p.Met1549Val }\end{array}$ \\
\hline CTNNB1 & $\begin{array}{c}\text { catenin beta } 1 \\
\text { known as: CTNNB, MRD19, armadillo }\end{array}$ & $3 p 22.1$ & $\begin{array}{l}\text { somatic: } \\
\text { S45F p.Ser45Phe } \\
\text { S45P p.Ser45Pro } \\
\text { S33C p.Ser33Cys }\end{array}$ \\
\hline ARMC5 & $\begin{array}{l}\text { armadillo repeat containing } 5 \\
\text { known as: } \mathrm{AIMAH} 2\end{array}$ & $16 \mathrm{p} 11.2$ & $\begin{array}{l}\text { germline: } \\
\text { R898W p.Arg989Trp } \\
\text { P826H p.Pro826His } \\
\text { F14Y p.Phe14Tyr } \\
\text { L156F p.Leu156Phe } \\
\text { I170V p.lle170Val } \\
\text { G323A p.Gly323Ala } \\
\text { P507L p.Pro507Leu } \\
\text { T643M p.Thr643Met } \\
\text { G798A p.Gly798Ala }\end{array}$ \\
\hline
\end{tabular}

patients with APAs to about $40 \% \cdot{ }^{19-21}$ Female patients are more often carriers of these mutations than male, which is estimated up to $56-68 \%$ of females in different reports..$^{20,23,25,26}$ Aldosterone producing adenomas (APAs) carrying $K C N J 5$ gene mutation were usually larger than APAs without mutation. ${ }^{20,23}$ Furthermore, carriers of these mutations had higher excretion of aldosterone and lower serum potassium level than non-carriers. ${ }^{23}$ 


\section{ATP1A1 and ATP2B3}

Beuschlein et al. in 2013 performed an exome sequencing of material derived from 9 patients with APA and wild type of Kir3.4 channel, and identified the presence of somatic mutations in other genes - ATP1A1 and ATP $2 B 3 .{ }^{27}$ In larger material, derived from 308 patients with APA, they confirmed the presence of the ATP1A1 and ATP $2 B 3$ mutations in $6.8 \%$ of cases. ${ }^{27}$ There was no simultaneous presence of both mutations in any case.

The ATP1A1 gene is located on chromosome 1p21 and encodes the alpha- 1 subunit $\mathrm{Na}^{+} / \mathrm{K}^{+}$ATPase. The pump is composed of 10 transmembrane segments labeled from M1 to M10. As previously mentioned, it is involved in maintaining a negative membrane resting potential (Fig. 2). Mutated ATPase has a lower affinity to potassium ions. It passively transports sodium and potassium ions into the cell, which results in the depolarization of the membrane, opening of the voltage-gated calcium channels and an increase in the intracellular calcium concentration all without angiotensin II action. ${ }^{27}$ The $1^{\text {st }}$ mutation described by Beuschlein et al. was related to the segment M1 of ATPase - p.Leu104Arg (L104R), the $2^{\text {nd }}$ to the segment M4 - p.Val332Gly (V332G), and then deletion in p.Phe100_ Leu104del was identified. These genetic changes lead to a rupture of glutamic acid at position 334, a key area for the proper binding of the sodium and potassium ions. ${ }^{27} \mathrm{Williams}$ et al. identified another point mutation in ATP1A1 - p.Gly99Arg (G99R), and estimated the prevalence of the ATP1A1 mutant in about $6.3 \%$ of patients. ${ }^{21}$ Another confirmed $A T P 1 A 1$ mutation was a substitution p.GluGluThrAla963Ser in the segment M9. ${ }^{28}$ Moreover, Åkerström et al. reported 6 novel ATP1A1 mutations and determined the frequency of their occurrence at a similar level $-6.1 \% .^{26}$

The ATP2B3 gene is located on chromosome $\mathrm{X}$ and encodes $\mathrm{Ca}^{2+}$ ATPase, a member of the same type ATPases family as the $\mathrm{Na}^{+} / \mathrm{K}^{+}$ATPase. It is similarly composed of 10 transmembrane segments. The $\mathrm{Ca}^{2+}$ ATPase plays an important role in the maintenance of intracellular calcium homeostasis being responsible for the removal of calcium ions from the cell's cytoplasm. Two mutations of $\mathrm{Ca}^{2+}$ ATPase in the key area for calcium ion binding have been described: p.Leu425_Val426del, p.Val426_pVal427del. ${ }^{27}$ Their presence concerns the M4 transmembrane segment of the pump and results in the improper binding of calcium ions, which impairs the function of the pump.

Further studies by Scholl et al., Åkerström et al., Dutta et al. and Murakami et al. led to the identification of further ATP2B3 mutations including: c.1281_1286delGGCTGT, p.Arg428-Val429del, V426G_V427Q_A428_L433del, p.Val424_Leu425del. 22,26,29,30

It has been observed that (ATP1A1 and $A T P 2 B 3)$ mutants had a higher membrane level of depolarization than the cells without mutation. Both $\mathrm{Na}^{+} / \mathrm{K}^{+}$ATPase and $\mathrm{Ca}^{2+}$ ATPase mutations occurred more frequently in male than female patients. ${ }^{27}$ The general prevalence of these mutations in patients with APAs was estimated to about 3.0\%.22,26

\section{CACNA1D}

The point mutation in the CACNA1D gene may lead to the development of hyperaldosteronism as well. ${ }^{24,26,28,31}$ CACNA1D gene is located on chromosome 3p14.3 and encodes the calcium ion channel $\mathrm{Ca}_{\mathrm{v}} 1.3$ belonging to the L-type voltage-dependent calcium channel family. It is composed of 4 homologous elements, each consisting of 6 membrane-spanning segments (referred to as S1 to S6). Subsequent mutations of the CACNA1D: p.Gly403Arg, p.Ile770Met, p.Ile750Met, p.Phe747Leu, p.Arg990His, p.Pro1336Arg, p.Val259Asp, V748I were identified by Wang et al., Åkerström et al., Azizan et al. and Scholl et al. ${ }^{24,26,28,31}$ The mutations lead to the opening of the calcium channels with the lower membrane potential, which results in earlier calcium current influx, an increase in the intracellular calcium concentration and aldosterone production. The incidence of these mutations in different studies range from $3.0-7.8 \%$. They occur more often in male than in female patients. ${ }^{26,31}$ It has been shown that dihydropyridine calcium channel blockers are weak inhibitors of $\mathrm{Ca}_{\mathrm{v}} 1.3$ so that they are considered to be effective therapy in APA patients carrying CACNA1D mutations. ${ }^{31}$

There is also data of somatic mutations in CTNNB1 gene in PHA patients, primarily found in some solid cancers. ${ }^{28,31}$ CTNNB1 encodes for beta catenin 1 involved in signal transduction pathways of cell growth regulation, stem cells and embryonic development. It is located on chromosome 3p22.1. ${ }^{32}$ Mutations in CTNNB1 seem to be rare in APAs. ${ }^{28,31,32}$ Some authors hypothesize they are gender specific. ${ }^{33}$

\section{Germline mutations and familial hyperaldosteronism}

The basis of familial hyperaldosteronism (FH) are germline mutations in genes encoding ion channels and enzymes involved in aldosterone synthesis. Mutations responsible for the excessive production of aldosterone in the familial hyperaldosteronism type I, III and IV have been identified, while the gene responsible for the FH type II still remains undetected. ${ }^{34}$

Familial hyperaldosteronism type I (FH1), known as glucocorticoid-remediable aldosteronism (GRA) affects about $1 \%$ of patients with PHA. ${ }^{1}$ It is characterized by severe hypertension of early-onset, often developing before the age of 20. Its molecular basis was described by Lifton et al. in $1992 .{ }^{14}$ The essence of FH1 is the phenomenon of a crossing over between 5 ' promoter region of the 11 beta-hydroxylase and 3' region of the aldosterone synthase, which are situated very closely to chromosome 8 . As a result of a chimeric gene creation, aldosterone secretion occurs in the zona fasciculata of the adrenal cortex, becomes the subject of ACTH action and shows a characteristic circadian rhythm similarly to cortisol. In the FH1 treatment, small doses of dexamethasone are effective, reducing blood aldosterone level reduction, and normalizing blood pressure. ${ }^{1}$ 
Familial hyperaldosteronism type II (FH2), first described by Gordon et al. in 1991, is considered to affect up to $6 \%$ of patients with PHA. ${ }^{34,35}$ Phenotypically, patients with FH2 do not differ significantly from patients with sporadic PHA. ${ }^{34}$ Familial hyperaldosteronism type II is mainly characterized by a positive family history of primary aldosteronism in at least 2 first-degree relatives. ${ }^{1}$ So far, no gene responsible for the development of the disease has been determined. The linkage to the region $7 \mathrm{p} 22$ is also considered, but the data is controversial and requires further confirmation. ${ }^{36,37}$ There are opinions that it could be a result of familial accumulation of sporadic PHA as well. ${ }^{34}$

The first mention of another possible familial hyperaldosteronism, type III (FH3), came in 2008 from Geller et al., who described the case of a father and 2 daughters suffering from severe hypertension of early onset. It was accompanied by deep hypokalemia, massive hypertrophy of both adrenal glands, and high urine concentrations of steroid hybrids 18-oxocortisol and 18-hydroxycortisol, but with no response to dexamethasone treatment, which distinguished them completely from FH1. ${ }^{38}$ The direct pathogenesis of the syndrome was unknown until Choi et al. described a similar case of a family. They performed sequencing of the KCNJ5 gene and identified a germline heterozygous point mutation - T158, which allowed to distinguish a new familial hyperaldosteronism type III (FH3). ${ }^{17}$ The mutation involves threonine - T158 lying in the area of potassium ion filter selectivity and results in the loss of its selectivity. This leads to an increased sodium inward current, cell membrane depolarization and the opening of the voltage-gated calcium channels. Calcium mobilization activates a cascade of calcium signaling pathways resulting in constantly increased aldosterone production and autonomous proliferation of glomerulosa cells. ${ }^{17}$ Scholl et al. identified the germline KCNJ5 mutations of: G151R and G151E, while another one - I157S - was described by Charmandari et al. ${ }^{39,40}$ It was observed that the G151R and T158A mutation carriers are characterized by severe aldosteronism escalating with age, a significant adrenal hyperplasia, and poor response to treatment with spironolactone. On the other hand, patients carrying the G151E mutation do not have adrenal hyperplasia, the disease has early onset, but a mild course; they also better respond to treatment with spironolactone. According to Scholl et al., this may be a result of increased apoptosis of cells with this type of mutation. ${ }^{39}$

Very recently, Scholl et al. performed an exome sequencing in 40 unrelated patients with early onset of hypertension by the age of 10 and hyperaldosteronism. ${ }^{41}$ They revealed the same heterozygous mutation in the CACNA1H gene - M1549V in 5 (12.5\%) subjects. It allowed us to identify a new type IV of familial aldosteronism (FH4). The CACNA1H gene is located on chromosome 16p13.3 and encodes the $\alpha 1$ subunit of the T-type voltage dependent calcium channel $\mathrm{Ca}_{\mathrm{v}} 3.2$. The CACNA1H mutation results in impaired channel activation and slower inactivation, which consequently leads to increased intracellular $\mathrm{Ca}^{2+}$ concentration and aldosterone production. ${ }^{41}$

Consecutive studies among patients with primary aldosteronism phenotype revealed germline mutations in CACNA1D gene. CACNA1D encodes for L-type voltage dependent calcium channel $\mathrm{Ca}_{\mathrm{v}} 1.3$. Two point germline mutations: p.Gly403Asp and p.Ile770Met in the CACNA1D gene were present in 2 of 100 tested patients with early onset of $\mathrm{PH} .{ }^{31}$ Because the representation (2\%) of this germline mutation was too small, new familial phenotype of primary aldosteronism has not been determined. Interestingly, patients who carried these mutations presented numerous additional symptoms besides hypertension, such as cerebral palsy, cortical blindness, seizures, spastic quadriplegia, neuromuscular disorders or mental retardation. It may suggest a much broader impact of these mutations on other cells and tissues, especially since it is known that beside adrenal glomerulosa cells the CACNA1D expression was detected in other tissues like heart, neurons, cochlear hair cells, muscle cells, and others. ${ }^{31,42}$

In patients with PHA the ARMC5 genetic alterations were also identified. ${ }^{43}$ This tumor-suppressor gene was originally found in cortisol-producing macronodular adrenal hyperplasia. ARMC5 gene is located on chromosome $16 \mathrm{p} 11.2$ and is a member of the ARM (armadillo/ beta-catenin-like repeat) superfamily. The ARM-repeat is a 42 amino acids motif repeated tandemly. ${ }^{44}$ It is implicated in protein-protein interactions. In 56 PHA patients Zilbermint et al. identified 12 germline ARMC5 mutations in 20 unrelated and 2 related patients (39.3\%), especially African Americans. ${ }^{43}$ Recently, Mulatero et al. described 18 successive ARMC5 mutations in Caucasian patients. ${ }^{45}$ There is little available data; however, germline ARMC5 variants might be associated with PHA.

\section{Polymorphisms of genes encoding ion channels}

Given the association between the KCNJ5 gene mutations and primary aldosteronism, establishing the relationship between genetic polymorphisms in KCNJ5 and susceptibility to PHA in hypertensive population seems to be important. Murthy et al. suggested a possible link of PHA with the E282Q polymorphism, which was found in 12 out of 251 patients with PHA (it is about $5 \%) \cdot{ }^{46} \mathrm{Li}$ et al. evaluated some associations of the rs3740835 (C/A) and rs2604204 (A/C) KCNJ5 polymorphisms with unilateral and bilateral aldosteronism. The study included 1,043 hypertensive patients (83 with unilateral aldosteronism, 142 with bilateral aldosteronism, and 818 with essential hypertension). Researchers showed a possible link between rs3740835 (C/A) polymorphism and unilateral hyperaldosteronism, but did not find the relationship with respect to bilateral hyperaldosteronism. The association between the rs2604204 (A/C) polymorphism and 
either unilateral or bilateral hyperaldosteronism has not been confirmed. ${ }^{47}$

So far, a small number of studies does not allow us to establish a unique link between polymorphisms of the KCNJ5 gene and primary aldosteronism. Analysis of the KCNJ5 polymorphisms, and possibly of the other above-mentioned genes, e.g. CACNA1D, requires further research.

\section{Mutations and polymorphisms in aldosterone synthase gene}

Aldosterone synthase, the key enzyme involved in the last step of aldosterone synthesis, is encoded by the CYP11B2 gene. The association between $C Y P 11 B 2$ polymorphisms and excessive production of aldosterone and hypertension have been investigated. However, the data is divergent about the relationship between aldosterone synthase gene polymorphisms and hyperaldosteronism.

There are 2 common polymorphisms of the CYP11B2 gene: $\mathrm{C}$ to $\mathrm{T}$ substitution in position -344 and a variant of intron 2. The first polymorphism C-344T is located in the promoter area of aldosterone synthase and is related to steroidogenic factor binding protein (SF-1) involved in the regulation of CYP11B2 transcription. The second polymorphism, a variant of intron 2 , is also known as a conversion allele because of the transference of the part of intron 2 of CYP11B1 to CYP11B2. ${ }^{48}$

A possible association between $\mathrm{C}-344 \mathrm{~T}$ polymorphism and serum aldosterone level in normotensive patients was described by Paillard in 1999. It was suggested that the presence of the allele $\mathrm{T}$ is associated with a higher level of serum aldosterone. ${ }^{49}$

In 2007 Sokooian et al. performed a meta-analysis concerning the linkage of the C-344T polymorphism with primary hypertension. They analyzed the data from 19 studies (mainly case-control and cohort studies) involving 5,343 patients with primary hypertension and 5,882 individuals from the control group. This analysis showed a relationship between $-344 \mathrm{C}$ allele (CC genotype) with a $17 \%$ reduction of the risk of hypertension compared to homozygous genotype $\mathrm{TT}^{50} \mathrm{~A}$ further meta-analysis performed by Li et al., based on 29 studies $(8,482$ case and 8,560 controls) of the Chinese population, also suggested a significant association of C-344T polymorphism with hypertension. ${ }^{51}$ On the contrary, the study of Chen et al. did not confirm the association of this polymorphism with increased susceptibility to hypertension. ${ }^{52}$ The latter meta-analysis consisted of 18 studies (4,739 case and 3,793 controls).

Inglis et al. evaluated the relationship between the CYP11B2 polymorphisms and increased secretion of aldosterone in patients with hormonally active adrenal adenoma. The frequency of the $344 \mathrm{~T}$ allele and conversion allele differed significantly in APA patients when compared to the healthy control group. However, these researchers did not find statistical significance in the frequency of these alleles in the material from APAs in relation to the control genomic DNA. It is assumed that these genotypes may rather predispose people to an increased aldosterone production than to tumor growth. ${ }^{53}$ Jia et al. performed a meta-analysis of available case-control studies to assess the relationship between $C Y P 11 B 2$ polymorphisms and primary hyperaldosteronism. They analyzed 7 studies concerning T-344C substitution (which were attended by 621 case and 1,027 controls) and 3 studies of the A2718G polymorphism (327 case and 336 controls). The study group consisted of patients with idiopathic aldosteronism (IHA) or aldosterone producing adenoma (APA), while patients with essential hypertension or without hypertension were included in the control group. The researchers found a relationship between $\mathrm{T}-344 \mathrm{C}$ polymorphism (recessive model) with idiopathic aldosteronism, but did not observe a similar relationship between patients with adrenal adenomas. There was no sufficient evidence and data to establish the relationship with the A2718G polymorphism and increased risk of PHA. Their study suggests that the $-344 \mathrm{C}$ allele may be associated with a lower risk of developing idiopathic primary aldosteronism. ${ }^{54}$

The association between transcription rate of CYP11B2 gene and its own polymorphism T-1651C was studied. McManus et al. found that the transcriptional repressor APEX1 binds the $-1651 \mathrm{~T}$ allele stronger than the $-1651 \mathrm{C}$ allele, which results in reduced CYP11B2 expression and consequently lower aldosterone production. The authors also reported a correlation between the variant $-1651 \mathrm{~T}$ and lower excretion rates of aldosterone metabolites. ${ }^{55}$

\section{Summary}

Primary hyperaldosteronism is an important clinical problem, considering its high prevalence among hypertensive subjects and unequivocally increased cardiovascular risk. Elucidating the pathophysiology and improving diagnostics of the disease is of great concern. Widely conducted genetic studies have shed new light on the pathogenesis of PHA, classifying primary hyperaldosteronism as a channelopathy. There is a significant frequency of somatic and germline mutations in ion channel encoding genes in PHA patients. The data is still inconsistent. In fact, there is numerous evidence of a predicted significance available in the literature; however, there is still a lack of a so-called "game changer" bringing the final conclusion for therapeutic and, hopefully, preventive strategies for the whole population. The possible link between gene polymorphism of aldosterone synthase or ion channels and both primary hypertensive disease and primary aldosteronism requires further research. The genetic investigation hopes to find new solutions for the future. Identifying carriers of potentially prognostic genetic variants would not only contribute to a more precise diagnosis, but to the development of a specific, new treatment as well. 


\section{References}

1. Funder JW, Carey RM, Mantero F, et al. Case detection, diagnosis, and treatment: An endocrine society clinical practice guideline. J Clin Endocrinol Metab. 2016;101:1889-1916.

2. Fagugli RM, Taglioni C. Changes in the perceived epidemiology of primary hyperaldosteronism. Int J Hypertens. 2011;2011:162804.

3. Rossi GP, Bernini G, Caliumi C, et al. A prospective study of the prevalence of primary aldosteronism in 1,125 hypertensive patients. J Am Coll Cardiol. 2006;48:2293-2300.

4. Conn JW. Primary aldosteronism, a new clinical syndrome. J Lab Clin Med. 1955;45:3-17.

5. Milliez P, Girerd X, Plouin PF, Blacher J, Safar ME, Mourad JJ. Evidence for an increased rate of cardiovascular events in patients with primary aldosteronism. J Am Coll Cardiol. 2005;45:1243-1248.

6. Fallo F, Veglio F, Bertello C, et al. Prevalence and characteristics of the metabolic syndrome in primary aldosteronism. J Clin Endocrinol Metab. 2006;91:454-459.

7. Weber KT, Brilla CG. Pathological hypertrophy and cardiac interstitium. Fibrosis and renin-angiotensin-aldosterone system. Circulation. 1991;83:1849-1865.

8. Stowasser M, Sharman J, Leano R, et al. Evidence for abnormal left ventricular structure and function in normotensive individuals with familial hyperaldosteronism type I. J Clin Endocrinol Metab. 2005;90:5070-5076.

9. Sechi LA, Fabio A, Bazzocchi M, Uzzau A, Catena C. Intrarenal hemodynamics in primary aldosteronism before and after treatment. J Clin Endocrinol Metab. 2009;94:1191-1197.

10. Freel EM, Mark PB, Weir RAP, et al. Demonstration of blood pressureindependent noninfarct myocardial fibrosis in primary aldosteronism: A cardiac magnetic resonance imaging study. Circ Cardiovasc Imaging. 2012;5:740-747.

11. Staermose S, Marwick TH, Gordon RD, Cowley D, Dowling A, Stowasser M. Elevated serum interleukin 6 levels in normotensive individuals with familial hyperaldosteronism type 1. Hypertension. 2009;53:31-33.

12. Bernini G, Galetta F, Franzoni F, et al. Arterial stiffness, intima-media thickness and carotid artery fibrosis in patients with primary aldosteronism. J Hypertens. 2008;26:2399-2405.

13. Connell JMC, Davies E. The new biology of aldosterone. J Endocrinol. 2005;186:1-20.

14. Lifton RP, Dluhy RG, Powers M, et al. A chimaeric 11 beta-hydroxylase/aldosterone synthase gene causes glucocorticoid-remediable aldosteronism and human hypertension. Nature. 1992;355:262-265.

15. Hattangady NG, Olala LO, Bollag WB, Rainey WE. Acute and chronic regulation of aldosterone production. Mol Cell Endocrinol.2012;350:151-162.

16. Guagliardo NA, Yao J, Hu C, Barrett PQ: Minireview: Aldosterone biosynthesis: Electrically gated for our protection. Endocrinology. 2012;153:3579-3586.

17. Choi $\mathrm{M}$, Scholl UI, Yue $\mathrm{P}$, et al. $\mathrm{K}^{+}$channel mutations in adrenal aldosterone-producing adenomas and hereditary hypertension. Science. 2011;331:768-772.

18. Mulatero $\mathrm{P}$, Tauber $\mathrm{P}$, Zennaro MC, et al. KCNJ5 mutations in European families with nonglucocorticoid remediable familial hyperaldosteronism. Hypertension. 2012;59:235-240.

19. Azizan $E A B$, Murthy $M$, Stowasser $M$, et al. Somatic mutations affecting the selectivity filter of KCNJ5 are frequent in 2 large unselected collections of adrenal aldosteronomas. Hypertension. 2012;59:587-591.

20. Åkerström T, Crona J, Delgado Verdugo A, et al. Comprehensive resequencing of adrenal aldosterone producing lesions reveal three somatic mutations near the KCNJ5 potassium channel selectivity filter. PLoS One. 2012;7:1-7.

21. Williams TA, Monticone S, Schack VR, et al. Somatic ATP1A1, ATP2B3, and KCNJ5 mutations in aldosterone-producing adenomas. Hypertension. 2014;63:188-195.

22. Scholl UI, Healy JM, Thiel A, et al. Novel somatic mutations in primary hyperaldosteronism are related to the clinical, radiological and pathological phenotype. Clin Endocrinol (Oxf). 2015;83:779-789.

23. Zheng FF, Zhu LM, Nie AF, et al. Clinical characteristics of somatic mutations in Chinese patients with aldosterone-producing adenoma. Hypertension. 2015;65:622-628.

24. Wang B, Li X, Zhang X, et al. Prevalence and characterization of somatic mutations in Chinese aldosterone-producing adenoma patients. Medicine (Baltimore). 2015;94:e708.
25. Zennaro $M C$, Jeunemaitre $X$, Boulkroun S. Integrating genetics and genomics in primary aldosteronism. Hypertension. 2012;60:580-588.

26. Åkerström T, Willenberg HS, Cupisti K, et al. Novel somatic mutations and distinct molecular signature in aldosterone-producing adenomas. Endocr Relat Cancer. 2015;22:735-744.

27. Beuschlein F, Boulkroun S, Osswald A, et al. Somatic mutations in ATP1A1 and ATP2B3 lead to aldosterone-producing adenomas and secondary hypertension. Nat Genet. 2013;45:440-442.

28. Azizan EA, Poulsen $H$, Tuluc $P$, et al. Somatic mutations in ATP1A1 and CACNA1D underlie a common subtype of adrenal hypertension. Nat Genet. 2013;45:1055-1060.

29. Dutta RK, Welander J, Brauckhoff M, et al. Complementary somatic mutations of KCNJ5, ATP1A1, and ATP2B3 in sporadic aldosterone producing adrenal adenomas. Endocr Relat Cancer. 2014;21:3-6.

30. Murakami M, Yoshimoto T, Minami I, et al. A novel somatic deletion mutation of ATP2B3 in aldosterone-producing adenoma. Endocr Pathol. 2015;26:328-333.

31. Scholl UI, Goh G, Stölting G, et al. Somatic and germline CACNA1D calcium channel mutations in aldosterone-producing adenomas and primary aldosteronism. Nat Genet. 2013;45:1050-1054.

32. CTNNB1 catenin beta 1. ncbi.nlm.nih.gov https://www.ncbi.nlm.nih. gov/gene/1499. Updated June 10, 2018. Accessed June 13, 2018.

33. Dutta RK, Soderkvist P, Grimm O. Genetics of primary hyperaldosteronism. Endocr Relat Cancer. 2016;23:R437-R454.

34. Mulatero P, Tizzani D, Viola A, et al. Prevalence and characteristics of familial hyperaldosteronism: The PATOGEN study (Primary aldosteronism in TOrino-GENetic forms). Hypertension. 2011;58:797-803.

35. Gordon RD, Stowasser M, Tunny TJ, Klemm SA, Finn WL, Krek AL. Clinical and pathological diversity of primary aldosteronism, including a new familial variety. Clin Exp Pharmacol Physiol. 1991;18:283-286.

36. So A, Duffy DL, Gordon RD, et al. Familial hyperaldosteronism type II is linked to the chromosome 7p22 region but also shows predicted heterogeneity. J Hypertens. 2005;23:1477-1484.

37. Carss KJ, Stowasser M, Gordon RD, O'Shaughnessy KM. Further study of chromosome 7p22 to identify the molecular basis of familial hyperaldosteronism type II. J Hum Hypertens. 2011;25:560-564.

38. GellerDS, Zhang J, WisgerhofMV, Shackleton C, Kashgarian M, Lifton RP. A novel form of human mendelian hypertension featuring nonglucocorticoid - remediable aldosteronism. J Clin Endocrinol Metab. 2008;93:3117-3123.

39. Scholl UI, Nelson-Williams C, Yue P, et al. Hypertension with or without adrenal hyperplasia due to different inherited mutations in the potassium channel KCNJ5. Proc Natl Acad SciUSA. 2012;109:2533-2538.

40. Charmandari E, Sertedaki A, Kino T, et al. A novel point mutation in the KCNJ5 gene causing primary hyperaldosteronism and earlyonset autosomal dominant hypertension. J Clin Endocrinol Metab. 2012;97:1532-1539.

41. Scholl Ul, Stolting G, Nelson-Williams C. Recurrent gain of function mutation in calcium channel CACNA1H causes early-onset hypertension with primary aldosteronism. Elife. 2015;4:e06315. doi: 10.7554/eLife.06315

42. CACNA1D Gene. genecards.org. https://www.genecards.org/cgi-bin/ carddisp.pl?gene=CACNA1D. Accessed July 27, 2016.

43. Zilbermint $M$, Xekouki $P$, Faucz FR, et al. Primary aldosteronism and ARMC5 variants. J Clin Endocrinol Metab. 2015;100:E900-909.

44. ARMC5 armadillo repeat containing 5. ncbi.nlm.nih.gov. https:// www.ncbi.nlm.nih.gov/gene/79798. Updated June 3, 2018. Accessed June 13, 2018.

45. Mulatero P, Schiavi F, Williams TA, et al. ARMC5 mutation analysis in patients with primary aldosteronism and bilateral adrenal lesions. J Hum Hypertens. 2016;30:374-378.

46. Murthy M, Xu S, Massimo G, et al. Role for germline mutations and a rare coding single nucleotide polymorphism within the KCNJ5 potassium channel in a large cohort of sporadic cases of primary aldosteronism. Hypertension. 2014;63:783-789.

47. Li N, Shi C, Li H, et al. Association of KCNJ5 gene rs3740835(C/A) and rs2604204(A/C) polymorphism with unilateral and bilateral primary aldosteronism. Chinese J Med Genet. 2014;31:233-237.

48. White PC, Slutsker L. Haplotype analysis of CYP11B2. Endocr Res. 1995; 21:437-442.

49. Paillard F, Chansel D, Brand E, et al. Genotype-phenotype relationships for the renin-angiotensin-aldosterone system in a normal population. Hypertension. 1999;34:423-429. 
50. Sookoian S, Gianotti TF, González CD, Pirola CJ. Association of the C-344T aldosterone synthase gene variant with essential hypertension: A meta-analysis. J Hypertens. 2007;25:5-13.

51. LiW, Liu C. The-344C/T polymorphism in the CYP11B2 gene is associated with essential hypertension in the Chinese. JRAAS. 2014;15:150-155.

52. Chen J, Jing J, Tan H, Song M, Yu S, Huang L. Lack of association of CYP11B2-344C/T polymorphism with essential hypertension: A meta-analysis. Int J Clin Exp Med. 2015;8:9162-9167.
53. Inglis GC, Plouin PF, Friel EC, Davies E, Fraser R, Connell JMC. Polymorphic differences from normal in the aldosterone synthase gene (CYP11B2) in patients with primary hyperaldosteronism and adrenal tumour (Conn's syndrome). Clin Endocrinol (Oxf). 2001;54:725-730.

54. Jia $M$, Zhang $H$, Song $X$, et al. Association of CYP11B2 polymorphisms with susceptibility to primary aldosteronism: A meta-analysis. Endocr J. 2013;60:861-870.

55. McManus F, Sands W, Diver L, et al. APEX1 regulation of aldosterone synthase gene transcription is disrupted by a common polymorphism in humans. Circ Res. 2012;111:212-219. 